\title{
SPHERICAL HARMONIC ANALYSIS OF EQUALIZATION IN A REVERBERANT ROOM
}

\author{
Terence Betlehem and Thushara D. Abhayapala \\ National ICT Australia, Department of Telecommunications Engineering \\ RSISE, Australian National University \\ Canberra, ACT, 0200, Australia \\ Email: [Terence.Betlehem, Thushara.Abhayapala]@anu.edu.au
}

\begin{abstract}
In this paper, we investigate the performance of acoustic equalization in reverberant environments. We first highlight an efficient general representation of a sound field using spherical harmonics. We then use this representation to develop a concise closed-form expression for robustness of equalization to sensor movement. This expression is used (i) to characterize equalization performance for a general class of non-isotropic sound fields and (ii) to quantify the improvements to equalizer robustness that can be obtained by using a directional microphone. The approach used here does not use any of the assumptions of statistical acoustics, but instead exploits the inherent properties of a sound field as described by the wave equation.
\end{abstract}

\section{INTRODUCTION}

A problem of hands-free telephony is acquiring undistorted speech in reverberant environments when a microphone cannot be located near the source. A solution is to use acoustic equalization, where signal distortion is removed with an appropriate inverse filter.

Unfortunately if the source and sensor positions are not fixed, acoustic equalization is difficult. The sound field varies greatly from point to point in a typical room [1]. Even a change in the source or microphone position of a few tenths of a wavelength creates a large variation in the room channel response and large degradation in equalized output $[2,3,4]$. Techniques have been suggested to combat this robustness problem $[5,6,7]$.

The multi-channel case proposed in [7] shows promise. However, as the robustness analysis in this case was based on the single-channel analysis of Radlovic̀ et al. [2], results are restricted to the assumptions of statistical room acoustics and a first order approximation only valid in moderately reverberant rooms. Further, the mean square error criterion used in [2] is overly-conservative as it is sensitive to the time delay of the equalizing filter.

In this paper, we derive an expression for a new performance criterion, not prone to the above problems. This criterion describes the robustness of magnitude response equalization in any sound field, and with a microphone of arbitrary directivity pattern. To do this, we exploit the modal decomposition of a sound field.

Below we present a deterministic approach. This approach is sophisticated enough to capture the important effects of the geometric parameters of the sound field, but is simple enough to yield a concise closed-form expression and permits understanding and generalization of geometry on performance.

\section{MODAL DECOMPOSITION OF A SOUND FIELD}

Denote the frequency domain signal received at an omnidirectional sensor at position $\boldsymbol{x}$ as $f(\boldsymbol{x} ; k)$ where $k \triangleq \omega / c$ is the wave number, $\omega$ is angular frequency and $c$ is the speed of sound in air. Define a spherical region $\Omega \in \mathbb{R}^{3}$ centered about the origin that excludes all sound sources. A general representation of the sound field inside $\Omega$ that obeys the Helmholtz wave equation is [8]:

$$
f(\boldsymbol{x} ; k)=\sum_{n, m} \alpha_{n m}(k) j_{n}(k x) Y_{n}^{m}(\hat{\boldsymbol{x}})
$$

where the summation $\sum_{n, m}$ denotes $\sum_{n=0}^{\infty} \sum_{m=-n}^{n}$, $\alpha_{n m}(k)$ are coefficients representing the wave field, $j_{n}(\cdot)$ are the spherical Bessel functions, $x \triangleq\|\boldsymbol{x}\|, \hat{\boldsymbol{x}} \triangleq \boldsymbol{x} /\|\boldsymbol{x}\|$ and $Y_{n}^{m}(\cdot)$ are the spherical harmonic functions

$$
Y_{n}^{m}(\hat{\boldsymbol{x}}) \triangleq \sqrt{\frac{2 n+1}{4 \pi} \frac{(n-m) !}{(n+m) !}} P_{n}^{m}(\cos \theta) e^{i m \phi},
$$

$\theta$ and $\phi$ are the elevation and azimuthal angles of $\hat{\boldsymbol{x}}$ respectively, and $P_{n}^{m}(\cdot)$ are the associated Legendre functions of the first kind. Spherical harmonic functions form an orthonormal set spanning the unit spherical shell $\mathbb{S}^{2}=\{\boldsymbol{x}$ : $\|\boldsymbol{x}\|=1\}$ and satisfy the orthogonality property,

$$
\int_{\mathbb{S}^{2}} Y_{n}^{m}(\hat{\boldsymbol{x}}) \overline{Y_{n^{\prime}}^{m^{\prime}(\hat{\boldsymbol{x}})}} d s(\hat{\boldsymbol{x}})=\delta_{m m^{\prime}} \delta_{n n^{\prime}}
$$


where $\overline{(\cdot)}$ is the complex conjugation operator and $d s(\hat{\boldsymbol{x}})$ is the spherical surface element at $\hat{\boldsymbol{x}}$. The basis functions $j_{n}(k x) Y_{n}^{m}(\hat{\boldsymbol{x}})$ are known as modes.

Equation (1) provides an efficient representation of a sound field. Due to the bandpass character of the spherical Bessel functions, it specifies the field over a spherical region centered about the origin with the minimum number of parameters [9].

\section{SOUND FIELD IN A ROOM}

In this section, we provide a framework for describing the sound field in a reverberant room.

\subsection{Direct and Reverberant Fields}

Separate the field $f(\boldsymbol{x} ; k)$ into two parts, a direct field $f_{D}(\boldsymbol{x} ; k)$ and reverberant field $f_{R}(\boldsymbol{x} ; k)$, with corresponding field coefficients $\alpha_{n m}^{(D)}$ and $\alpha_{n m}^{(R)}$ respectively,

$$
f(\boldsymbol{x} ; k)=f_{D}(\boldsymbol{x} ; k)+f_{R}(\boldsymbol{x} ; k) .
$$

By linearity of (1) then,

$$
\alpha_{n m}=\alpha_{n m}^{(D)}+\alpha_{n m}^{(R)} .
$$

For brevity, the dependence on $k$ has been suppressed. Let the source be placed at position $y \in \mathbb{R}^{3} / \Omega$. The direct field component is that part of the field arriving directly without reflection,

$$
f_{D}(\boldsymbol{x} ; k)=\frac{e^{-i k\|\boldsymbol{y}-\boldsymbol{x}\|}}{4 \pi\|\boldsymbol{y}-\boldsymbol{x}\|} .
$$

The direct field coefficients $\alpha_{n m}^{(D)}$ can be found with the spherical harmonic expansion of the direct part [8],

$$
\begin{aligned}
\frac{e^{-i k\|\boldsymbol{y}-\boldsymbol{x}\|}}{4 \pi\|\boldsymbol{y}-\boldsymbol{x}\|}= & -i k \sum_{n, m} h_{n}^{(2)}(k y) \overline{Y_{n}^{m}(\hat{\boldsymbol{y}})} \times \\
& j_{n}(k x) Y_{n}^{m}(\hat{\boldsymbol{x}}), \quad y>x
\end{aligned}
$$

where $h_{n}^{(2)}(\cdot)$ is the spherical Hankel function of the second kind. Comparing (1) with (6),

$$
\alpha_{n m}^{(D)}=-i k h_{n}^{(2)}(k y) \overline{Y_{n}^{m}(\hat{\boldsymbol{y}})} .
$$

The reverberant field is specified completely through the reverberant field coefficients $\alpha_{n m}^{(R)}$, defined by

$$
f_{R}(\boldsymbol{x} ; k)=\sum_{n, m} \alpha_{n m}^{(R)} j_{n}(k x) Y_{n}^{m}(\hat{\boldsymbol{x}}) .
$$

The relative magnitude of the direct and reverberant field components is measured by the direct-to-reverberant ratio. This is defined as the ratio of the energy density of the direct part to the energy density of the reverberant field. From
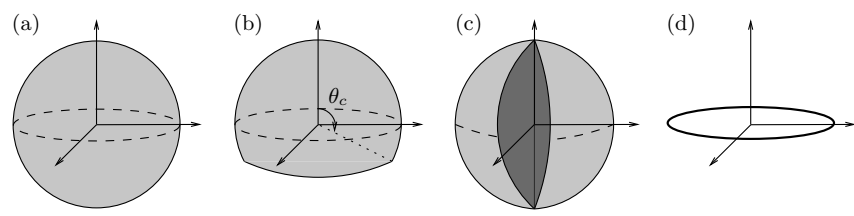

Fig. 1. Configurations of reverberant sources around a sphere. (a) Isotropic shell. (b) Conical sector with half cone angle $\theta_{c}$. (c) Spherical slice. (d) Circular ring.

synthesis equation (1), the direct-to-reverberant ratio at the origin reduces to,

$$
\gamma_{0} \triangleq\left|\frac{f_{D}(\mathbf{0} ; k)}{f_{R}(\mathbf{0} ; k)}\right|^{2}=\left|\frac{\alpha_{00}^{(D)}}{\alpha_{00}^{(R)}}\right|^{2} .
$$

\subsection{Reverberation Modelling}

An arbitrary wave field inside a source-free region can be generated by a set of sources arranged on the region boundary [10]. This motivates us to define of the class of wave fields generated by distributing attenuated copies of the source, here called reverberant sources over a spherical shell with radius $R$. Defining the unit sphere $\mathbb{B} \subset \mathbb{S}^{2}$, the reverberant field $f_{R}(\boldsymbol{x} ; k)$ is calculated by:

$$
f_{R}(\boldsymbol{x} ; k)=\sigma_{R} \int_{\mathbb{B}} \frac{e^{-i k\|\boldsymbol{x}-R \hat{\boldsymbol{v}}\|}}{4 \pi\|\boldsymbol{x}-R \hat{\boldsymbol{v}}\|} d s(\hat{\boldsymbol{v}}),
$$

where $\sigma_{R}$ controls the energy density of the reverberation and $R$ is the radius of the shell. Applying (6), we can show the reverberant field coefficients $\alpha_{n m}^{(R)}$ to be

$$
\alpha_{n m}^{(R)}=\kappa_{n} \int_{\mathbb{B}} \overline{Y_{n}^{m}(\hat{\boldsymbol{v}})} d s(\hat{\boldsymbol{v}}),
$$

where $\kappa_{n} \triangleq-i k \sigma_{R} h_{n}^{(2)}(k R)$.

Below are presented several geometric configurations of reverberant sources shown in Fig. 1. The associated coefficients $\alpha_{n m}^{(R)}$ are summarized in Table 1.

\subsubsection{Isotropic Shell}

For the isotropic shell, we equally distribute a continuum of reverberant sources over a spherical shell (Fig. 1(a)). This field is composed of only one mode, $j_{0}(k x) Y_{0}^{0}(\hat{\boldsymbol{x}})$. Here the reverberation arrives at the sensor with equal contributions from each direction. The isotropic shell is hence the deterministic analog to the 3D isotropic field [11], and describes the reverberation best in rectangular rooms with homogeneous wall parameters. 


\begin{tabular}{|c|c|}
\hline Configuration & $\alpha_{n m}^{(R)}$ \\
\hline Sphere & $4 \pi \kappa_{n} \Lambda_{0}^{0} \delta_{m 0} \delta_{n 0}$ \\
Conical sector & $2 \pi \kappa_{n} \Lambda_{n}^{0} \sin \theta_{c} P_{n}^{-1}\left(\cos \theta_{c}\right) \delta_{m 0}$ \\
Spherical slice & $2 \Delta \phi \kappa_{n} \Lambda_{n}^{m} j_{0}(m \Delta \phi) \int_{-1}^{1} P_{n}^{m}(u) d u$, \\
Circular ring & $2 \pi \kappa_{n} \Lambda_{n}^{0} P_{n}(0) \delta_{m 0}$ \\
\hline
\end{tabular}

Table 1. Reverberant field coefficients for various geometries; $\kappa_{n} \triangleq-i k \sigma_{R} h_{n}^{(2)}(k R) ; \Lambda_{n}^{m} \triangleq \sqrt{\frac{2 n+1}{4 \pi} \frac{(n-m) !}{(n+m) !}}$.

\subsubsection{Spherical Sector}

Uniformly distribute a continuum of reverberant sources over the conical sector $\mathbb{B}_{\text {cone }}=\{(R, \theta, \phi): 0<\theta<$ $\left.\theta_{c}, 0<\phi<2 \pi\right\}$ (Fig. 1(b)) and the spherical slice $\mathbb{B}_{\text {slice }}=$ $\{(R, \theta, \phi): 0<\theta<\pi,-\Delta \phi<\phi<\Delta \phi\}$ (Fig. 1(c)). The spherical sector is useful for describing non-spherically symmetric fields where the reverberation comes only from a certain range of directions. Fields with directional character provide a simple model for room inhomogeneities.

\subsubsection{Circular Ring}

A circular ring is the deterministic equivalent of the $2 \mathrm{D}$ isotropic case, for microphones restricted to the plane of the ring. Let the ring be centered about the origin in the $x y$ plane $\mathbb{B}_{\text {ring }}=\left\{(R, \theta, \phi): \theta=\frac{\pi}{2}, 0<\phi<2 \pi\right\}$ (Fig. 1(d)). The circular ring model describes the reverberant field best in rooms with a highly sound-absorbing floor and ceiling.

\section{DUAL INTERPRETATION}

Consider a microphone with a directional response $D(\hat{\boldsymbol{v}} ; k)$ depending on direction $\hat{\boldsymbol{v}}$ and wave number $k$, with spherical harmonic expansion,

$$
D(\hat{\boldsymbol{v}} ; k)=\sum_{n, m} \xi_{n m}(k) Y_{n}^{m}(\hat{\boldsymbol{v}})
$$

In the above reverberation model, we can show that for $x \ll$ $R$ the output of this sensor is given by

$$
f_{R}(\boldsymbol{x} ; k)=\sigma_{R} \int_{\mathbb{B}} D(\hat{\boldsymbol{v}} ; k) \frac{e^{-i k\|\boldsymbol{x}-R \hat{\boldsymbol{v}}\|}}{4 \pi\|\boldsymbol{x}-R \hat{\boldsymbol{v}}\|} d s(\hat{\boldsymbol{v}}) .
$$

Comparing with (10), we see the output signal of the directional sensor,

$$
D_{\mathbb{B}^{\prime}}(\hat{\boldsymbol{v}} ; k)= \begin{cases}1, & \hat{\boldsymbol{v}} \in \mathbb{B}^{\prime}, \\ 0, & \text { otherwise, }\end{cases}
$$

in a isotropic shell is equal to that of an omnidirectional sensor in a shell with geometry parameter equal to $\mathbb{B} \cap \mathbb{B}^{\prime}$. Further, a microphone with arbitrary $D(\hat{\boldsymbol{v}} ; k)$ in a field generated by the isotropic shell geometry can be shown to be equivalent to an omnidirectional microphone in a field with coefficients $\kappa_{n} \xi_{n m}$.

\section{ROBUSTNESS OF EQUALIZATION}

We now develop a measure of robustness of magnitude response equalization to changes in sensor position. To quantify this, define the equalizer error criterion $\epsilon$ as follows. Let $H(k)$ be the frequency response of a zero-forcing equalizer attached to a sensor. Choosing the origin at the sensor, $H(k)=1 / f(\mathbf{0} ; k)$. If the sensor is then moved to position $\boldsymbol{r}$, the source-to-sensor transfer function becomes $G(k)=f(\boldsymbol{r} ; k)$. The magnitude square error in equalizer output due to movement of the sensor is $|G(k) H(k)|^{2}-1$. Define the average equalization error $\epsilon(r ; k)$ as:

$$
\epsilon(r ; k)=\frac{1}{4 \pi} \int_{\mathbb{S}^{2}}\left(\left|\frac{f(\boldsymbol{r} ; k)}{f(\mathbf{0} ; k)}\right|^{2}-1\right) d s(\hat{\boldsymbol{r}}) .
$$

This error measures the average error in equalizer output for a movement of distance $r \triangleq\|\boldsymbol{r}\|$. Exploiting the modal expansion, we derive an expression for $\epsilon$ in any sound field $f(\boldsymbol{r} ; k)$ given by the synthesis formula (1).

\section{Robustness Expression}

The average equalization error due to movement of a sensor by a distance $r$ in a sound field $f(\boldsymbol{r} ; k)$ is given by:

$$
\epsilon(r ; k)=\sum_{n, m}\left|\frac{\alpha_{n m}}{\alpha_{00}}\right|^{2}\left[j_{n}(k r)\right]^{2}-1,
$$

where $\alpha_{n m}$ are the modal coefficients of the sound field.

This expression is obtained by substituting (1) into (13), and applying orthogonality property (2). Although the sensor has been placed at the origin to simplify analysis, this by no means limits the usefulness of the criterion.

\section{EXAMPLES}

We now evaluate the average equalizer error for several field geometries of Section 3. We quantify equalization performance with the zone of equalization, the spherical region in which $\epsilon$ does not exceed $-10 \mathrm{~dB}$. The parameters used were $c=342 \mathrm{~m} / \mathrm{s}, y=3 \mathrm{~m}, R=8 \mathrm{~m}$ and $\omega=2 \pi \mathrm{kHz}$.

Fig. 2(a) shows a plot of the error curves for an omnidirectional sensor in the field created by an isotropic shell of reverberation, for several values of $\gamma_{0}$. Here the 'reverberation only' case predicts a similar robustness curve to that in [2]. The 'reverberation only' zone of equalization has a radius of $0.1 \lambda$.

Fig. 2(b) compare the error curves for the field created by the conical sector of half cone angle $\theta_{c}$ and spherical slice of width $2 \Delta \phi$. Angle $\Delta \phi$ was chosen to make $\gamma_{0}$ the same as a corresponding conical sector case. The direct source has been positioned in the center of the conical sector and spherical slice (at $(0,0, y)$ and $(y, 0,0)$ respectively). 


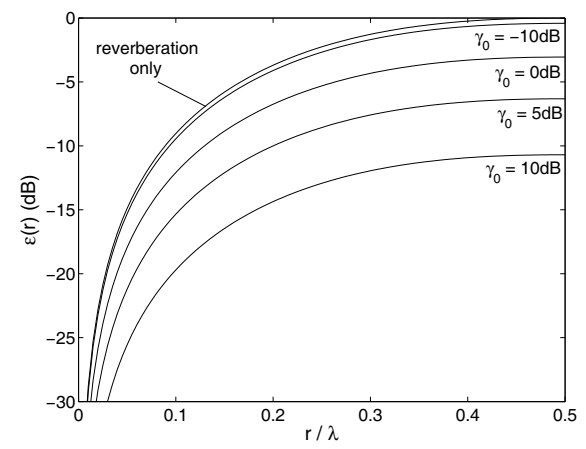

(a)

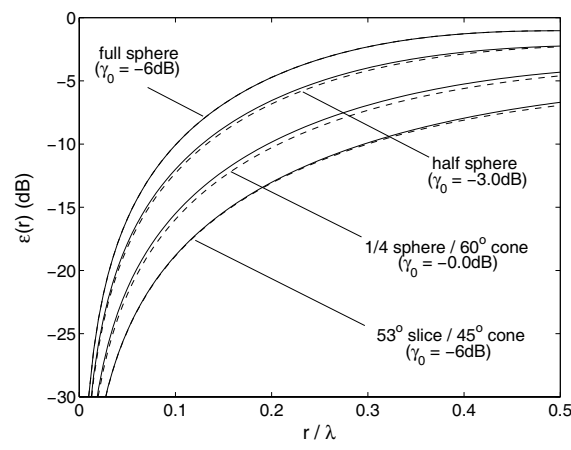

(b)

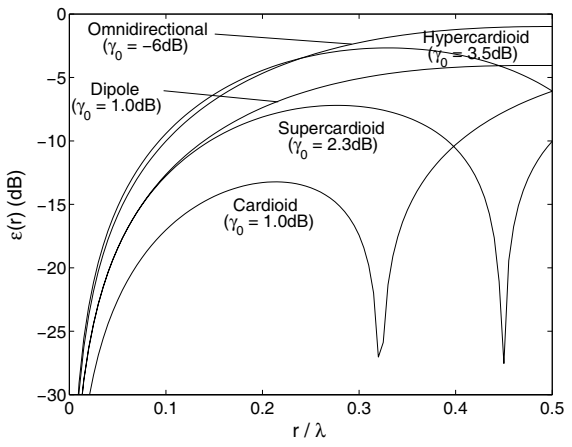

(c)

Fig. 2. Average equalization error for (a) the isotropic shell, (b) the conical sector and spherical slice and (c) various second order microphone designs (in this case $R=12.49 \mathrm{~m}$ ) in an isotropic shell.

The conical sector and spherical slice are shown to have approximately equal equalizer robustness. However with different $R$ and $y$, the conical sector is on average better. For small $\theta_{c}$ the sources on the conical sector are more tightly concentrated, producing a field with a greater coherence.

Fig. 2(b) allows estimation of the improvement attainable by cutting a proportion of the reverberation in a room. For the half sphere configuration the radius of the zone of equalization increases by $30 \%$, and for the $1 / 4$ sphere, by $110 \%$.

Finally, we exploit the dual interpretation to quantify the robustness for the second order directional microphones designs of [12] in an isotropic shell. Fig. 2(c) shows improvement can be gained by increasing microphone directivity.

\section{CONCLUSION}

Modal analysis has been used to develop a concise closedform expression for the robustness of magnitude response equalization to sensor movement in any sound field. We characterized the robustness in several sound fields to show the dependence of the zone of equalization on the geometric parameters of the field. A dual interpretation of a sound field has extended analysis to directional sensors, showing a dependence of performance on sensor directivity. ${ }^{1}$

\section{REFERENCES}

[1] J. Mourjopoulos, "On the variation and invertibility of room impulse response functions," J. Sound Vib., vol. 102, no. 2, pp. 217-228, 1985.

[2] B. D. Radlovic, R. C. Williamson, and R. A. Kennedy, "Equalization in an acoustic reverberant environment: Robustness results," IEEE Trans. Signal Processing, vol. 8, no. 3, pp. 311-319, 2000.

\footnotetext{
ideas.
}

[3] P.A. Nelson, F. Orduna-Bustamante, and H. Hamada, "Multichannel signal processing techniques in the reproduction of sound," J. Aud. Eng. Soc., vol. 44, no. 11, pp. 973 - 989, 1996.

[4] L.D. Fielder, "Analysis of tradional and reverberationreducing methods of room equalization," J. Aud. Eng. Soc., vol. 51, no. 1/2, pp. 3-26, 2003.

[5] J. Mourjopoulos, "Digital equalization of room acoustics," J. Aud. Eng. Soc., vol. 42, pp. 884 - 900, 1994.

[6] S. Bharitkar and C. Kyriakakis, "A cluster centroid method for room response equalization at multiple locations," in Proc. IEEE Workshop on the Applicatons of Signal Processing to Audio and Acoustics, 2001.

[7] F. Talantzis and D. B. Ward, "Robustness of multichannel equalization in an acoustic reverberant environment," J. Acoust. Soc. Amer., (accepted).

[8] D. Colton and R. Kress, Inverse acoustic and electromagnetic scattering theory, Springer-Verlag, Berlin, 1992.

[9] H. M. Jones, R. A. Kennedy, and T. D. Abhayapala, "On dimensionality of multipath fields: Spatial extent and richness," in Proc. of ICASSP, Orlando, 2002.

[10] R. A. Kennedy, T. D. Abhayapala, and T. S. Pollock, "Generalized herglotz wave functions for modelling wireless nearfield multipath scattering environments," in Proc. of ICASSP, Hong Kong, 2003, vol. IV.

[11] H. Kuttruff, Room Acoustics, Applied Science Publishers, London, second edition, 1979.

[12] G. W. Elko, "Superdirectional microphone arrays," pp. 181-237. Kluwer Academic Publishers, 2000, Chapter 10. 